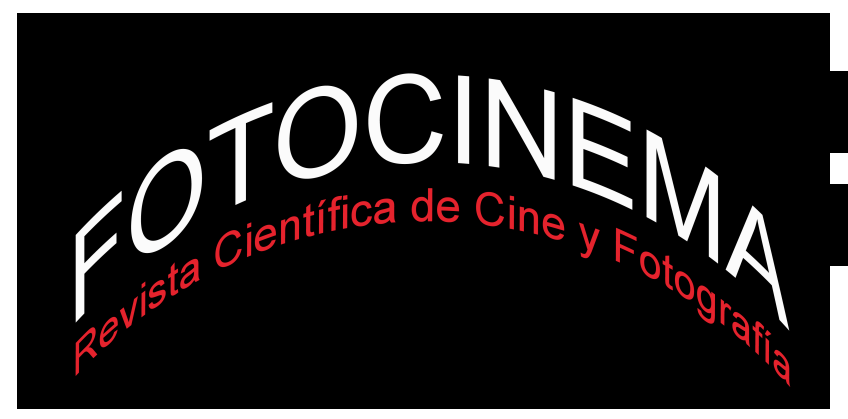

Recibido 13-09-2017 / Aceptado 15-12-2017 Publicado 29/01/2018

\title{
JULIA ROBERTS ET CHARLIZE THERON DANS MIRROR, MIRROR ET SNOW WHITE AND THE HUNTSMAN : DEUX MÉCHANTES REINES EN CONCURRENCE, SOUS L'INFLUENCE DE L'HÉRÖ̈NE DE WALT DISNEY
}

\section{JULIA ROBERTS AND CHARLIZE THERON IN MIRROR, MIRROR AND SNOW WHITE AND THE HUNTSMAN: TWO COMPETING WICKED QUEENS, UNDER THE INFLUENCE OF WALT DISNEY'S HEROINE}

\section{Résumé:}

En 2012, Hollywood a proposé deux nouvelles versions de Blanche Neige Mirror, Mirror de Tarsem Singh et Snow White and the Huntsman de Rupert Sanders. Si de nombreuses adaptations de ce conte ont été tournées, la référence reste la version animée de Disney (1937). Concurrence oblige, les films de Singh et Sanders doivent se démarquer l'un de l'autre en devant gérer la même difficulté : créer la surprise par rapport aux attentes fondées sur le souvenir du film de Disney. Ce point commun explique que, malgré leurs différences, les deux films offrent le rôle de la reine à leurs stars, Julia Roberts et Charlize Theron. Le lien entre l'œuvre de référence et ces nouvelles versions s'établit grâce à ce personnage qui, chez Disney, est le moins «cartoonesque » et révèle la dualité qui caractérise la création du héros d'animation, dans la mesure où sa représentation est déterminée par le jeu vocal de Lucille La Verne. Cette dualité se retrouve dans les interprétations de Roberts et Theron qui évoluent entre le cartoon et le " réalisme ». L'incarnation de la reine par les deux stars nous démontre combien la frontière entre personnages animés et personnages « réels » est poreuse.

\section{Arnaud Duprat de Montero \\ Université Rennes 2, Francia Arnaud.duprat@univ-rennes2.fr}

\begin{abstract}
:
In 2012, two new versions of Snow White came out of Hollywood - Tarsem Singh's Mirror, Mirror and Rupert Sanders's Snow White and the Huntsman. Although many adaptations of this tale have been released, Disney's animated version (1937) remains the reference. Due the obligations of competition, Singh and Sanders needed to ensure that each of their films stood out in relation to the other by dealing with the same difficulty: creating surprise in relation to expectations based on the Disney film. This common element explains why, despite their differences, the two films give their respective stars, Julia Roberts and Charlize Theron, the role of the queen. The link between the film which is seen as the benchmark and these new versions is revealed thanks to the character who, in the Disney film, is the least "cartoonish" and reveals the duality which characterises the creation of heroes in animated works, in that the way she is played is determined by Lucille La Verne's voice work. This duality can be found in the performances of Roberts and Theron who walk the line between cartoons and "realism". The embodiment of the queen by these two stars shows us how the porous the frontier between animated characters and "real" characters can be.
\end{abstract}




\title{
JULIA ROBERTS Y CHARLIZE THERON EN BLANCANIEVES \\ (MIRROR, MIRROR) Y BLANCANIEVES Y LA LEYENDA DEL \\ CAZADOR: DOS MALVADAS REINAS EN COMPETENCIA, BAJO LA \\ INFLUENCIA DE LA HEROÍNA DE WALT DISNEY
}

\begin{abstract}
Resumen:
En 2012, Hollywood propuso a los espectadores dos nuevas versiones de Blancanieves Blancanieves (Mirror, Mirror) de Tarsem Singh y Blancanieves y la leyenda del cazador (Snow White and the Huntsman) de Rupert Sanders- en el intervalo de unas pocas semanas. Si se rodaron numerosas adaptaciones de este cuento de los hermanos Grimm en varios países a lo largo de la historia del cine, la referencia ineludible sigue siendo hoy día la película de animación de Walt Disney, Blancanieves y los siete enanitos (Snow White and the Seven Dwarfs), estrenada en 1937. En una lógica de competencia, los filmes de Tarsem Singh y de Rupert Sanders deben distinguirse el uno del otro y al mismo tiempo resolver la misma dificultad: sorprender con respecto a las expectativas basadas en el recuerdo de la película de Walt Disney. Este punto común podría explicar el hecho de que, a pesar de sus diferencias radicales -el filme de Tarsem Singh se presenta como una comedia con reflejos bollywoodienses, mientras que el de Rupert Sanders propone un universo con una estética influenciada por la Heroic fantasy-Mirror, Mirror y Snow White and the Huntsman ofrecen el papel de la reina malvada a sus dos estrellas, Julia Roberts y Charlize Theron. El vínculo entre la obra de referencia y estas nuevas versiones se establece gracias a este personaje que, en la película de Walt Disney, es el menos " cartoonesco » y que da más realce a la dualidad que caracteriza la creación de un héroe de animación. Definida por el propio Walt Disney como una mezcla entre Lady Macbeth y el lobo feroz - del corto metraje Los tres cerditos (Three Little Pigs, Walt Disney, 1933)-, la reina fue creada gracias a la técnica de la rotoscopia - que consiste en filmar a actores reales y después en calcar las imágenes producidas - como fuente de inspiración, pero con distorsiones respecto a la realidad ya que el realismo de un personaje animado exige más velocidad y más movimentos. También fue creada gracias a la actriz Lucille La Verne cuya actuación vocal determinó su representación y animación en la pantalla. Volvemos a encontrar esta dualidad en las actuaciones de Julia Roberts y de Charlize Theron que, al tener que tomar en cuenta el recuerdo de la reina de Walt Disney, la otra actriz "competidora” y la expresión de su propia persona, se sitúan entre la tentación del cartoon y la del "realismo". La encarnación de la reina por las dos estrellas y los roces producidos bien demuestran cuánto, en el cine, la frontera entre personajes animados y personajes de carne y hueso es, al final, borrosa y porosa. Además, lo que aporta cada actriz -su actuación, su persona y la manera de hacer referencia a la heroína de Walt Disney- permite nuevas lecturas del cuento originario, hablándonos al mismo tiempo de la sociedad actual, y permite también poner de relieve el substrato psicanalítico del clásico de 1937, después de los análisis previos de Bruno Bettelheim, dedicados al cuento literario.
\end{abstract}

Palabras clave: Imagen real; cine de animación; adaptación; Star Studies; J. Roberts; C. Theron.

Keywords: Live Action; Animated Films; Adaptation; Star Studies; J. Roberts; C. Theron

Mots-clé: Prise de vue réelle; cinéma d'animation; adaptation; Star Studies; J. Roberts ; C. Theron. 
Cómo citar: Duprat de Montero, A. (2018). Julia Roberts et Charlize Theron dans Mirror, mirror et Snow white and the huntsman: deux méchantes reines en concurrence, sous l'influence de l'héroïne de Walt Disney. Fotocinema. Revista científica de cine y fotografía, $\mathrm{n}^{0}$ 16, pp. 289-305. Disponible: http://www.revistas.uma.es/index.php/fotocinema/ DOI: http://dx.doi.org/10.24310/Fotocinema.2017.voi15

\section{Introduction}

Si l'histoire du cinéma est, depuis ses origines, ponctuée par de nombreux personnages qui apparaissent de manière régulière sur les écrans de continents différents, multipliant ainsi les incarnations et les films mettant en scène leurs histoires, il est moins ordinaire de rencontrer dans le même pays et au même moment deux d'entre eux. Un exemple de cette situation nous a été donné en 2012 par Hollywood qui a proposé à seulement quelques semaines d'intervalle ${ }^{1}$ deux adaptations du conte Blanche Neige des frères Grimm, publié en 1812 : Mirror, Mirror de Tarsem Singh et Snow White and the Huntsman de Rupert Sanders. Le conte, du fait qu'il n'est lui-même que la version écrite d'une histoire aux origines inconnues et transmises oralement, avec des variations notables, de génération en génération à travers plusieurs pays, se prête idéalement au phénomène de la sérialité cinématographique en inscrivant ce dernier dans une évolution littéraire où les innovations font loi. Les films de Singh et Sanders ne dérogent pas à la règle. Ainsi, Mirror, Mirror se présente comme une comédie parodique aux teintes bollywoodiennes ${ }^{2}$, tandis que Snow White and the Huntsman propose un univers violent à l'esthétique influencée par l'Heroic Fantasy3. Un des rares points communs entre ces deux films est de réserver le rôle de la méchante reine à leurs stars: Julia Roberts et Charlize Theron. Ce rôle

1. Mirror, Mirror sortit aux États-Unis le 30 mars 2012 et Snow White and the Huntsman le 1 juin 2012.

2. Auteur de vidéoclips pour Vanessa Paradis, REM, Suzanne Vega... et de publicités, Tarsem Singh avait déjà réalisé trois longs métrages : The Cell (2000), The Fall (2006) et Immortals (2011).

3. Il s'agit du premier long-métrage du britannique Rupert Sanders. 
charismatique devient alors un des enjeux principaux de la concurrence entre les deux œuvres. Cette concurrence est également déterminée par le fait que, malgré les nombreuses adaptations cinématographiques du conte dont la plus ancienne remonte à 19024, la référence incontournable reste aujourd'hui encore le film de Walt Disney, Snow White and the Seven Dwarfs, sorti en 1937. Singh et Sanders doivent se démarquer l'un de l'autre en devant gérer la même difficulté : créer la surprise par rapport aux attentes des spectateurs fondées sur le souvenir du film de Disney. Dans le phénomène de la sérialité au cinéma, il est finalement assez fréquent qu'une œuvre en particulier s'affirme comme la référence : par exemple, de toutes les Anna Karénine, comment oublier encore aujourd'hui Greta Garbo ou Vivien Leigh? Cependant, le cas de ces nouvelles adaptations de Blanche Neige est intéressant dans la mesure où cette référence incontournable est ici un film d'animation. Ainsi, les incarnations de Julia Roberts et de Charlize Theron se voient déterminées par l'autre actrice concurrente et l'affirmation nécessaire de sa propre persona, certes, mais également par le souvenir de la méchante reine de Disney. Afin d'étudier le lien qui se crée ici entre personnage animé et actrices de chair et de sang, il s'agit, dans un premier temps, de revenir à l'œuvre de Disney et d'évaluer sa place intertextuelle dans les films de Singh et Sanders. Une fois ce cadre établi, nous nous intéresserons particulièrement à la création du personnage de la méchante reine chez Disney, de sa genèse à sa représentation à l'écran, tout en soulignant les caractéristiques communes aux interprétations de Julia Roberts et Charlize Theron. Enfin, dans la mesure où ces caractéristiques pourraient bien trouver un sens en s'inscrivant dans l'évolution de la persona de chacune, nous verrons comment ces deux nouvelles adaptations éclairent l'histoire

\footnotetext{
4. Dans son ouvrage consacré au film de Disney, J. B. Kaufman souligne qu'en 1902 «(..) motion-picture pioneer Siegmund Lubin had advertised a version of Snow White as part of a selection of fairy-tale films ». (Kaufman, 2012, p. 24). Kaufman recense également une adaptation de 1910 produite par les frères Pathé, La petite Blanche Neige, une version produite par la Powers Company en 1913, une version amateur de la Educational Films de Cleveland, finalement sortie en salles en 1916, puis celle de J. Searle Dawley pour la Paramount Film, avec Marguerite Clark dans le rôle-titre, également en 1916, que Walt Disney vit quand il était enfant. Enfin, Betty Boop endossa les habits de l'héroïne des frères Grimm dans un court-métrage de Fleischer/Paramount en 1933. (Kaufman, 2012, pp. 2426).
} 
fondatrice et constituent ainsi l'étape actuelle et cohérente d'un phénomène de réécriture perpétuelle.

\section{Cadre théorique et méthodologie}

Certains commentateurs (Gaudreault \& Marion, 2013; Massuet, 2014) ont vu l'apparition du numérique et ses nouveaux procédés - Motion Capture, Performance Capture - comme un dépassement d'un « cinéma-institution dont le territoire esthétique et définitionnel a été fondé entre autres sur une séparation technique entre cinéma en prises de vues réelles et cinéma d'animation » (Massuet, 2014, p. 2). Même si les deux nouvelles adaptations de Blanche Neige par Hollywood en 2012, ont recours dans certaines séquences aux effets spéciaux numériques, la comparaison inévitable avec le classique de Disney reprend cette «séparation technique» du «cinémainstitution » évoquée plus haut. L'objectif de mon travail est, en me centrant sur la représentation à l'écran d'un personnage en particulier - la méchante reine - d'interroger l'évidence de cette séparation. Avec les outils méthodologiques des études actorales et des Star Studies, ma démarche analytique vise à mettre en lumière la part « humaine » dans la création de la méchante reine de Disney - le jeu vocal de Lucille La Verne et la rotoscopie ainsi que l'influence de ce personnage animé dans le jeu et la persona de Julia Roberts et de Charlize Theron. S'il n'existe pas de publication universitaire sur les films de Sanders et Singh, mon article se situe dans la continuité des nouveaux ouvrages consacrés au classique de Disney, publiés essentiellement en 2012 à l'occasion des soixante-quinze ans du film (Kaufman, 2012 ; Smodin, 2012 ; Derisson, 2014), qui visaient à réévaluer son importance dans l'histoire du cinéma, au-delà des limites de sa simple identité générique - le cinéma d'animation.

\section{Deux nouvelles adaptations marquées par le souvenir du film de Walt Disney}

Commençons en rappelant la place qu'occupe Snow White and the Seven Dwarfs de Walt Disney dans l'histoire du cinéma. Bien qu'étant en effet le 
premier long-métrage d'animation hollywoodien à une époque où le dessin animé était cantonné au format du court, les risques encourus par ce film ne concernaient pas uniquement sa durée. Tout d'abord, quand a débuté la production du film en 1934, la représentation des êtres humains dans le cinéma d'animation était toujours problématique. La majorité d'entre eux restait sur le mode du cartoon et, dès qu'un plus grand réalisme était visé notamment par le studio Disney dans certaines Silly Symphonies comme The Goddess of Spring en 1934 -, le résultat restait encore trop caricatural et approximatif. De plus, beaucoup d'inconnues entouraient la future réception de l'œuvre : le public était-il prêt à suivre un dessin animé pendant quatrevingt minutes où des héros réalistes se mêleraient à d'autres personnages plus «cartoonesques»? Une héroïne animée était-elle capable d'effrayer comme devait le faire la méchante reine? Les spectateurs allaient-ils accepter que cette dernière soit une meurtrière ? De la même façon, le public allait-il trembler pour Blanche Neige quand le chasseur se précipiterait sur elle avec un couteau ?... Aujourd'hui nous le savons, le film a tellement bien fonctionné qu'il est vite devenu à sa sortie en décembre 1937 le plus gros succès de l'histoire avant d'être détrôné deux ans plus par Gone With the Wind (George Cukor, Victor Fleming, Sam Wood, 1939). Au fil de nombreuses ressorties en salles avant sa première édition en vidéo en 1994, la popularité de Snow White and the Seven Dwarfs ne s'est jamais démentie et éclipse aujourd'hui encore le conte des frères Grimm et ses nombreuses adaptations, animées ou non, qui jalonnent l'histoire du cinéma.

Ainsi, les références au film de Disney dans Mirror, Mirror et Snow White and the Huntsman sont nombreuses et, du fait que, dans le premier cas, des qualités humaines servent de prénoms aux nains - ce qui fut un apport de Disney à la version des frères Grimm - et que, dans le deuxième, la méchante reine est une sorcière aux pouvoirs surnaturels - ce qui n'est pas exploité dans le conte 5 - les films de Singh et Sanders pourraient même apparaitre comme des remakes non avoués de Disney ${ }^{6}$. Pour les deux réalisateurs, la

5. Il est juste question à un moment de « son art de sorcière ». (Grimm, 2012, p. 14).

6. L'influence de Disney sur ces deux films dépasse le seul cas de Snow White and the Seven Dwarfs. Si l'esthétique médiévale et le siège final du château, chez Sanders, évoquent 
démarche est la même : partir des attentes des spectateurs par rapport au souvenir de Snow White and the Seven Dwarfs pour jouer sur des effets de surprise. Ainsi, les moments d'anthologie de 1937, comme par exemple la pomme empoisonnée, ne constituent finalement que des épisodes secondaires - voire un simple clin d'œil dans Mirror, Mirror - pour donner plus d'importance à des épisodes ajoutés - le siège du château et la confrontation finale entre Blanche Neige et la reine chez Sanders, la lutte avec le dragon et les retrouvailles avec le père chez Singh - qui assurent toute l'originalité de ces nouvelles versions et les inscrivent dans l'air du temps.

Nous remarquerons à ce sujet que la préoccupation de l'âge et la recherche de la jeunesse éternelle y prennent le pas sur l'obsession de la beauté sans rivale. Julia Roberts s'amuse ainsi de son âge en prétendant être de la même génération que le prince, et se livre même à une séance de soins esthétiques parodiant, dans le respect du ton général du film, les techniques actuelles, tandis que Charlize Theron, plus effrayante, se plonge intégralement dans un bain mystérieux censé prolonger sa jeunesse quand elle ne peut se nourrir de jeunes filles séquestrées dans son donjon7. Ces films se placent donc sous le signe du féminin, voire du féminisme, dans la mesure où les reines entendent conserver la couronne du royaume face à deux Blanche Neige bien plus combatives que chez Disney. De plus, Snow White and the Huntsman apporte une explication à la méchanceté de l'héroïne : ayant connu la misère dans son enfance, elle fut rejetée par son premier mari au profit d'une femme plus jeune. Dès lors, elle entretient sa beauté et sa jeunesse par la magie et

Sleeping Beauty (1959), nous retrouvons le duel final face au dragon à la fin de Mirror, Mirror. Dans ce même film, la chaumière des nains ressemble au foyer des enfants perdus dans Peter pan (1953), le château rappelle celui de Cinderella (1950) - il y est d'ailleurs question d'un bal en vue d'un mariage - et l'animation est présente dans la première séquence résumant les origines de Blanche Neige et dans le Praxinoscope qu'y manipule la reine.

7. De ce fait, on ne peut éviter de penser à la méchante reine du film de Terry Gilliam, The Brothers Grimm (2005) qui détient également un miroir magique et nourrit sa jeunesse des jeunes enfants kidnappés au village voisin. Le fait que cette reine soit incarnée par Monica Bellucci, autre beauté classique également égérie de Dior comme Charlize Theron, renforce ce rapprochement. Enfin, si les pratiques de ces reines relèvent du vampirisme, rappelons que dans le conte des frères Grimm, la reine est cannibale : quand le chasseur lui rapporte le foie et les poumons d'un marcassin, en faisant croire à cette dernière qu'il s'agit de ceux de Blanche Neige, la reine « les fit bien assaisonner et cuire : et la méchante femme crut manger la chair et le sang de Blanche Neige » (Grimm, 2012, p. 10). 
les utilise pour se venger des hommes - en l'occurrence le père de Blanche Neige - et dérober leur pouvoir politique. Nous retrouvons le même schéma dans Mirror, Mirror cette fois-ci sur le mode humoristique. Les rapports de force qui en découlent avec les belles-filles déterminent le casting des deux films : alors que Blanche Neige est incarnée par des actrices en devenir - Lily Collins et Kristen Stewart -, le rôle de la reine est donc proposé à deux stars confirmées qui déplacent les attentes des spectateurs et l'idée de concurrence entre les deux opus, de l'héroïne à la marâtre.

\section{Deux nouvelles reines tiraillées entre le souvenir de l'héroïne de Disney et la persona de deux stars}

L'interprétation de la méchante reine par Julia Roberts et Charlize Theron s'inscrit dans la même démarche duelle, entre prise en compte nécessaire de l'héroïne de Disney et revendication d'une originalité qui passe par la pleine expression de sa persona pour se distinguer de l'actrice concurrente. La dualité caractérise également la méchante reine de 1937. Si, dans les premières esquisses du personnage, celle-ci devait être une reine très «cartoonesque », ridiculement autoritaire et souffrant d'une surcharge pondérale - tout comme la reine de Cœur d'Alice in Wonderland quatorze ans plus tard (1951) -, dès une note de production du 22 octobre 1934, Walt Disney la présente à la croisée « de Lady Macbeth et du Grand Méchant Loup " (Cité in Finch, 1999, p. 51) de son grand succès Three Little Pigs (1933). Cette tension entre l'humain et le cartoon se confirme tout au long de sa création9. Rappelons que l'animation du personnage s'est vue déterminée par l'interprétation vocale de l'actrice Lucille La Verne qui incarna l'héroïne aussi bien quand elle est reine qu'après sa transformation en sorcière, toute

8. Si une autre note de production évoque également les masques de l'illustrateur Art Deco Wadislaw Theodor Benda (Kaufman, 2012, p. 78), Kaufman relève la grande ressemblance entre le costume de la reine et celui que porte Helen Gahagan dans le film de la RKO She (Lansing C. Holden, Irving Pichel, 1935). Robin Allan et Brian Sibley, quant à eux, citent comme source possible d'inspiration la statue-colonne de la cathédrale de Naumburg, en Allemagne, représentant Uta, épouse du margrave de Meissen (Allan \& Sibley, 1999, p. 54). Enfin, nombreux ont été les commentateurs à souligner la ressemblance troublante entre la reine et Joan Crawford, même si aucune note de production ne cite la star hollywoodienne.

9. Créée par Joe Grant, la reine fut animée par Art Babbitt, responsable de l'animation de Goofy, des trois petits cochons et des souris dans The Country Cousin, tandis que la sorcière fut attribuée à Norm Ferguson qui avait déjà animé Pluto et surtout le Grand Méchant Loup. 
la différence étant assurée, dans ce dernier cas, par l'absence de son dentier (Johnston \& Thomas, 1995, p. 54). L'influence humaine sur l'animation de l'héroïne ne s'arrêta pas là puisque les animateurs du studio utilisèrent la technique du rotoscope qui consiste à "décalquer» des photogrammes d'acteurs filmés. Cependant, cette technique ne servit que de source d'inspiration car les animateurs remarquèrent que, paradoxalement, les mouvements d'un personnage animé, s'ils reprennent fidèlement ceux issus du rotoscope, ne sont pas crédibles $^{10}$ : le personnage animé se doit tout d'abord d'être perpétuellement en mouvement, sinon il n'existe plus et redevient un simple dessin, et ses mouvements, pour être perçus comme réalistes, doivent être plus rapides et plus démonstratifs que ceux des humains ${ }^{11}$.

Ces tensions entre l'humain et le personnage animé sont présentes également chez les méchantes reines de Mirror, Mirror et Snow White and the Huntsman. Nous remarquerons les robes démesurées et très colorées portées par Julia Roberts dans le premier film, et surtout ses sautes d'humeur - on pense davantage à d'autres méchants disneyiens comme la reine déjà citée d'Alice in Wonderland, le capitaine Crochet de Peter Pan (1953)... - qui appellent un jeu très contrasté frôlant parfois la pantomime pour atteindre des effets comiques - citons notamment la scène de l'annonce de la mort de Blanche Neige aux cuisinières du château ${ }^{12}$-. La méchante reine de Charlize

10. Art Babbitt : « Je voudrais préciser que je n'ai jamais utilisé le rotoscope pour la reine. Il est vrai qu'on a d'abord tourné un film avec une actrice, observée ensuite sur la Moviola (sorte de visionneuse), mais après l'avoir étudiée, j'ai créé toute l'animation de tête sans revoir le film, et c'est une des bonnes raisons pour lesquelles je l'ai faite ». (Renaut, 1997, p. 18). « (...) study it and then put it away; and then animate your impression of what you have seen, keeping in mind that the timing, the spacing and the proportions are considerably different in live action. » (Holliss \& Sibley, 1987, p. 32).

11. Hamilton Luske, un des animateurs de Blanche Neige, explique : «Un personnage doit être exagéré. Il faut que ce soit plus intéressant que la vie ordinaire. Nos acteurs sont plus entraînés que les gens ordinaires. Si un personnage monte à cheval ou s'assoit sur une chaise, il faut que ce soit le plus simple et professionnel possible. Nos acteurs doivent être plus intéressants que vous et moi. Leur processus de pensée doit être plus rapide. Notre progression inintéressante d'une situation à l'autre doit être évitée ». De son côté, Walt Disney ajoute : « On a trouvé un truc qui consiste à rendre le dessin un peu flou, à l'allonger. Quand on bouge vite, l'action prend du mouvement, ce n'est pas la quantité de dessins, mais la manière dont ils sont faits. » Enfin, Frank Thomas, qui anima notamment la scène de la veillée funèbre, affirme que " si on ne fait pas bouger le personnage, il a l'air plat. Ça le tue et le message ne passe pas. » (Disney, 2009, audiocommentaires).

12. Ces mouvements appuyés évoquent les propos des animateurs de Snow White and the 
Theron se place plus particulièrement en correspondance avec l'héroïne de Disney par sa beauté froide et altière, ses costumes souvent sombres qui ne reprennent que partiellement les codes vestimentaires médiévaux - nous retrouvons le même jeu de déploiement des capes ou des robes longues -, sa droiture élancée qui détermine ses mouvements. Tout comme la reine de 1937, l'expression de ses sentiments passe alors essentiellement par des jeux de regards qui, du reste, en ne relevant que de la méchanceté ou de la douleur, respectent aussi bien les frères Grimm que Disney ${ }^{13}$. Enfin, ce lien se confirme car l'actrice prête son apparence à toutes les transformations numériques qui la font vieillir et rajeunir - parfois dans la douleur - en quelques secondes et dans un seul et même plan, chose possible en 1937 uniquement dans le cinéma d'animation et dont Disney tire profit lors de la célèbre scène de transformation.

Cependant, bien avant ce moment, la scène d'anthologie liée à la reine est, bien entendu, celle où elle se place face à son miroir magique. Cette séquence ne pouvait donc manquer dans ces deux nouveaux films, et c'est paradoxalement là où le souvenir de Disney est le plus fort, que la persona des deux actrices transparaît de manière incontournable. Remarquons tout d'abord que c'est le film de Sanders qui colle davantage au film de Disney, par le décor médiéval, la taille imposante du miroir et surtout la reprise de la célèbre question : "Who's the fairest one of all ? " Cependant, si le génie chez Disney est un visage aperçu dans le miroir, chez Sanders, il sort de l'objet pour venir se placer face à la reine et, en reprenant plus ou moins sa taille, former une silhouette dont le doré évoque la chevelure blonde de l'héroïne. Il apparaît alors comme son double - cette comparaison se confirme d'ailleurs quand la reine sort de son bain rajeunissant et que ses traits disparaissent sous le liquide - d'autant plus inquiétant qu'il est approximativement humain et non identifiable. Il est alors à l'image de la

Seven Dwarfs. Remarquons d'ailleurs que Julia Roberts effectue sans arrêt des mouvements, même minimes. Par exemple, tout comme la reine de Disney, elle tapote sans cesse du bout de ses doigts les accoudoirs de son trône.

13. Dans une note de production, Walt Disney précise que « her face and voice are almost always expressionless. Even when angry, or torturing her victims, her voice is low, cool, serene... Her eyes are usually veiled by heavy, half-closed lids. Thus, when she opens them suddenly, there is dramatic value in the movement ». (Kaufman, 2012, p. 78). 
personnalité véritable de l'héroïne, de sa monstruosité sous-jacente, toujours prête à ressurgir sous la beauté classique et de ce fait angoissante, d'où le jeu angoissé de l'actrice. Cette idée a caractérisé plusieurs rôles antérieurs de Charlize Theron dont le plus marquant reste celui de Monster (Patty Jenkins, 2003)14. Mirror, Mirror présente la même conception du génie. Là également, il peut se déplacer et n'est plus cantonné à un seul miroir, mais apparaît dans plusieurs. Néanmoins, en raison de la persona bien trempée de Julia Roberts, beaucoup moins ouverte à l'idée de transformation ou de performance, le génie a logiquement son apparence et cette reine s'inscrit dans l'évolution de cette persona. En étant excessive dans son comportement, volontiers teigne et prête à tout pour obtenir un homme qui ne lui correspond pas, ce nouveau personnage évoque une des héroïnes les plus célèbres de Julia Roberts, celle de My Best Friend's Wedding (P. J. Hogan, 1997). Si la persona de Charlize Theron se situe harmonieusement dans la continuité de la méchante reine de Disney, celle de Julia Roberts résiste à cette dernière et cette résistance se confirme dans son refus d'énoncer la célèbre question. Elle s'oppose ainsi à toute idée de rivalité physique et générationnelle avec sa belle-fille car ne voulant pas assumer une quelconque filiation, comme, à quelques exceptions près - Erin Brockovich (Steven Soderbergh, 2000), Fireflies in the Garden (Dennis Lee, 2011) ou Stepmom (Chris Columbus, 1998) -, Julia Roberts a évité les rôles de mères tout au long de sa carrière. Le ton parodique du film ne touche plus uniquement l'histoire de Blanche Neige, mais concerne également la persona de l'actrice.

\section{Deux démarches actorales qui éclairent de manière nouvelle le conte}

$\mathrm{Si}$, en créant un lien entre ce personnage de méchante reine et leur persona, Julia Roberts et Charlize Theron gèrent de manière opposée le souvenir de l'héroïne de Disney et réussissent à se distinguer par la même occasion l'une

14. Remarquons que le passé de cette héroïne, prostituée dès l'adolescence, qui décide un jour de tuer ses clients, entretient des ressemblances troublantes avec celui de la méchante reine de Sanders. 
de l'autre, leur démarche permet de nouvelles lectures du conte fondateur. Il est tout d'abord intéressant de relever que les deux actrices assument le personnage dans toutes ses transformations. Alors que la reine de Disney devient une autre personne avant de se rendre à la chaumière des nains, les héroïnes de Julia Roberts et Charlize Theron ne font que révéler physiquement leur âge véritable. Tandis que la laideur de la sorcière est à l'image de la véritable personnalité de la reine chez Disney, cette représentation du moi profond passe aujourd'hui par la vieillesse, comme une tare devant être cachée. Nous l'avons dit, l'obsession de la jeunesse qui en découle a des résonances actuelles évidentes. Cependant, les films de Sanders et Singh soulignent surtout ses conséquences désastreuses sur la filiation. Celles-ci sont déjà présentes chez les frères Grimm, même si le thème de la jeunesse n'est jamais explicite. Bruno Bettelheim, dans son célèbre ouvrage Psychanalyse des contes de fées, précise bien que

L'attitude de la reine devant son miroir rappelle le vieux thème de Narcisse, qui finit par se laisser engloutir par l'amour qu'il avait de lui-même. Ce sont les parents les plus narcissiques qui se sentent les plus menacés par la croissance de leur enfant. Celui-ci leur montre, en prenant de l'âge, qu'ils vieillissent. Tant que l'enfant est totalement dépendant, il continue, pour ainsi dire, de faire partie du père et surtout de la mère. Mais quand, mûrissant, il tend vers son indépendance, il est ressenti comme une menace, et c'est ce qui arrive à la reine de "Blanche Neige". (Bettelheim, 1976, p. 256)

L'évolution de la princesse relève en effet d'un parcours initiatique, souligné par le séjour chez les nains - dont la petite taille illustre, selon Bettelheim, leur « (incapacité) d'atteindre une virilité adulte » (Bettelheim, 1976, p. 252) et symbolise " une forme immature et pré-individuelle de conscience que Blanche Neige doit transcender » (Bettelheim, 1976, p. 264). Ce parcours, s'il relève du latent chez Disney au profit de la mise en scène d'une lutte entre le bien et le mal15, occupe le devant des histoires de Singh et Sanders. Dans les deux films, Blanche Neige est animée d'une conscience politique face à la gouvernance cruelle de la reine et entend bien récupérer la couronne de son

15. Karen Merritt : «Blanche-neige de Disney représente le monde d'un enfant au sein d'un monde naturel bienveillant et met ces éléments en opposition avec une ennemie tellement résolue et implacable qu'elle doit être détruite ». (Disney, 2009, audiocommentaires). 
père et s'inscrire ainsi dans sa filiation. Nous l'avons dit, l'opposition à la marâtre se réalise et les histoires s'achèvent avec l'entrée de la princesse dans l'âge adulte, en lien avec l'initiation sexuelle : la Blanche Neige de Singh est mariée par son père lui-même et abandonne sa longue chevelure pour un chignon et une frange plus adultes ${ }^{16}$, tandis que celle de Sanders est couronnée reine dans une robe rouge sang, à la symbolique sexuelle évidente. Cette filiation problématique pour la reine est plus complexe dans Snow White and the Huntsman que dans Mirror, Mirror où son refus est en lien avec l'attirance que ressent le personnage de Julia Roberts pour le prince charmant, ce qui relève, nous l'avons dit, de la persona de l'actrice, mais ce qui semble être également sous-entendu chez Disney puisque c'est après avoir surpris de sa fenêtre sa belle-fille avec le prince que la reine convoque finalement le chasseur ${ }^{17}$. Cependant, par la suite, elle ne cherche nullement à retrouver le prince et, tout comme dans le film de Sanders où le personnage de Charlize Theron entretient sa jeunesse comme une arme de séduction dont elle ne fait finalement rien depuis le meurtre du père de Blanche Neige, elle ne tire jamais profit de sa beauté qu'elle tente d'ailleurs de rendre la plus asexuée possible ${ }^{18}$. Cette ambiguïté dans le rapport à la jeune fille rend la démarche de la reine de Sanders malsaine, énigmatique et véritablement monstrueuse, en accord une fois encore avec la persona de l'actrice. En effet, même si la mère de la princesse apparaît dans la séquence inaugurale tout comme dans le conte des frères Grimm, un lien filial n'en existe pas moins entre la marâtre et la jeune fille : la reine s'appelle ici Ravenna alors qu'au début du film, il nous a été précisé que Blanche Neige naîtra avec des cheveux noirs comme les corbeaux - il est question d'ébène dans le conte et les trois gouttes de sang versées par la mère sur la neige trouvent un écho dans celles que verse la méchante reine sur l'armure de Blanche Neige lors de

16. Nous pensons bien sûr à Audrey Hepburn dans Roman Holidays (William Wyler, 1953) et Sabrina (Billy Wilder, 1954) où elle décide de couper ses cheveux comme un signe d'émancipation.

17. Dans le conte des frères Grimm, la rencontre entre Blanche Neige et le prince n'a lieu qu'à la fin, quand l'héroïne se trouve déjà dans son cercueil.

18. Nous retrouvons là certainement une réminiscence de Lady Macbeth quand elle demande aux esprits : « (...) unsex me here,/And fill me from the crown to the toe topful/Of direst cruelty! » William SHAKESPEARE, Macbeth, Act 1, scene 5, 41-43. 
l'affrontement final. La monstruosité de l'héroïne, en relevant de l'infanticide, illustre les vertus bienfaitrices du conte des frères Grimm auprès des enfants. En effet, Bruno Bettelheim a souligné que

la division de la mère en deux personnages (si caractéristique des contes de fées) une mère bonne - le plus souvent décédée - et une méchante marâtre, rend un grand service à l'enfant. C'est non seulement pour lui une façon de préserver en lui-même l'image d'une mère toujours bonne, quand la vraie mère ne l'est pas, c'est aussi pour l'enfant la possibilité d'être en colère contre cette méchante «marâtre » sans entacher la bienveillance de la vraie mère, qu'il considère comme une autre personne (Bettelheim, 1976, p. 95).

La confrontation à la reine s'inscrit alors dans le processus initiatique de la princesse et la tuer revient à «tuer la mère » pour atteindre l'âge adulte. Il est révélateur que, dans Snow White and the Huntsman, cette confrontation a lieu dans la salle du miroir et que, une fois la reine morte, le premier mouvement de Blanche Neige est de se regarder dans le miroir sans y trouver un génie, mais tout simplement son reflet ${ }^{19}$. Néanmoins, le film de Sanders ne renoue pas ainsi uniquement avec l'esprit du conte ${ }^{20}$, mais illustre également tout le contenu latent du film de Disney, malgré la volonté affichée de ce dernier, nous l'avons dit, de déplacer les lectures psychanalytiques possibles du conte vers un combat entre le bien et le mal. En effet, dès les premiers plans où un livre s'ouvre pour nous permettre de lire le début de l'histoire, il n'est aucunement question de la mère de Blanche Neige. Par la suite, la répétition de situations entre la reine et sa belle-fille semble tisser entre les deux femmes un lien secret. Lors de leurs premières scènes, leurs reflets interviennent - dans le miroir pour la reine, dans le puits pour

19. Concernant le miroir et son discours, Bettelheim écrit : «Le miroir magique semble parler avec la voix de la fille plutôt qu'avec celle de la mère. Habituellement, la toute petite fille pense que sa mère est la plus belle femme du monde, et c'est exactement ce que commence par dire le miroir à la reine. Mais, en grandissant, la petite fille pense qu'elle est beaucoup plus belle que sa mère, et c'est également ce que dit plus tard le miroir. Une mère peut s'effrayer quand elle se regarde dans un miroir ; elle se compare à sa fille et se dit en elle-même : "Ma fille est plus belle que moi." Mais, dans le conte, le miroir répond : "Elle l'est mille fois plus que vous" ; cette déclaration traduit bien l'exagération d'une adolescente qui amplifie ses avantages pour faire taire la petite voix intérieure qui veut la faire douter de sa supériorité ». (Bettelheim, 1976, p. 261).

20. Dans le conte des frères Grimm, cette filiation est d'autant plus ambiguë que, dans la première publication de 1812 , la reine était bel et bien la mère de Blanche Neige. Ce n'est que lors de la seconde édition de 1819 qu'elle devint la belle-mère de la princesse. 
Blanche Neige -; dès que le prince arrive, les deux héroïnes se trouvent derrière leurs fenêtres et ferment des rideaux rouges. Si la princesse semble vivre en osmose dans la nature et avec les animaux, il en va de même de la reine qui, dans son château, est accompagnée de rats ou d'un corbeau, avant d'être suivie dans la forêt par deux vautours. Par la suite, tandis que Blanche Neige se penche sur la marmite pour goûter la soupe préparée à l'attention des sept nains, la reine est dans son château au-dessus du chaudron où elle s'apprête à plonger la pomme dans le poison; en s'approchant de la chaumière, la sorcière est vue à travers une fenêtre comme Blanche Neige lors de son arrivée... Enfin, elles portent le même grand col baleiné blanc et leurs visages présentent le même teint et les mêmes lèvres rouge sang. Lors de la scène de transformation, le temps d'un instant, nous pouvons vérifier que, sous la guimpe, les cheveux de la reine sont aussi noirs que ceux de Blanche Neige ${ }^{21}$. Ce dernier élément est bien le seul qui crée une distance entre l'héroïne d'animation et l'incarnation de Charlize Theron. La chevelure blonde qui la féminise, révèle que la noirceur de l'héroïne est sous-jacente en elle, en accord avec la persona de l'actrice, mais elle révèle également tout le paradoxe de notre époque où la recherche de la perfection physique par les femmes ne relèverait plus uniquement d'une démarche narcissique, mais plutôt d'une condition nécessaire pour prendre le pouvoir dans un monde où la beauté féminine éveille la convoitise masculine et la tentation de transformer les femmes en objets de désir'22.

\section{Conclusion}

Si la monstruosité de la méchante reine de Charlize Theron offre ainsi une vision critique de la situation féminine, elle permet également une lecture de l'œuvre de Disney qui pourrait bien expliquer le fait que, parmi tous les films

21. Nous retrouvons ces correspondances chez Singh dans les robes des deux héroïnes. Lors de leur première scène, elles portent la même teinte beige. Pendant le bal, le chapeau-tête de cygne de Blanche Neige évoque le col en roue de paon de la reine. Par la suite, celle-ci alterne entre une robe bleue et une autre jaune. Ce sont ces deux couleurs qui composeront la robe de mariée de Blanche Neige.

22. Ceci est appuyé par l'épisode de la communauté exclusivement féminine où Blanche Neige et le chasseur trouvent refuge et où les femmes se sont délibérément défigurées pour échapper au désir masculin et être ainsi libres. Cette liberté est néanmoins synonyme d'une exclusion sociale que la méchante reine refuse. 
d'animation produits par le studio et tous leurs personnages de méchants, la reine demeure sans doute la plus effrayante auprès du jeune public. Ces nouvelles lectures, également présentes dans Mirror, Mirror, même si dans une moindre mesure, jouent sur une démarche alliant hommage et distanciation par rapport à l'œuvre de Disney. Dans la mesure où ces deux nouveaux films y relèvent en priorité le thème de la filiation problématique entre la méchante reine et sa belle-fille, ils sont révélateurs de notre société actuelle, mais également, à un niveau métacinématographique, de la propre filiation dans laquelle ils s'inscrivent. En effet, au sein de cette filiation, un classique âgé pourtant de quatre-vingts ans continue d'étouffer toutes les nouvelles versions du conte des frères Grimm qui l'ont suivi. De la même façon, il pouvait être a priori problématique aux acteurs de se confronter au souvenir de personnages animés. Cependant, dans le cas des nouvelles incarnations de la méchante reine par Julia Roberts et Charlize Theron, nous avons vu que l'opposition n'est jamais totale et que des liens se sont créés avec l'hérö̈ne de Disney, dans la mesure où la double nature animée et humaine de cette dernière appelle un jeu particulier chez les deux actrices, où l'influence de l'animation n'annule pas l'affirmation de leur persona. Tout en semblant revendiquer une filiation avec l'héroïne de Disney, leur persona, afin de se démarquer de l'autre actrice concurrente, n'en engendre pas moins des décalages et c'est précisément dans ces décalages qu'un nouvel éclairage sur l'œuvre de Disney est possible et permet aux deux films de dépasser le statut quelque peu stérile de remake ou de simple hommage. Des actrices de chair et de sang s'imposaient donc et en ce sens nous comprenons combien au cinéma la frontière entre animation et prise de vue réelle, est finalement floue.

\section{Références bibliographiques}

Allan, R \& Sibley, B. (1999). Walt Disney and Europe: European Influences on the Animated Feature Films of Walt Disney. London: John Libbey Cinema and Animation.

Bettelheim, B. (1976). Psychanalyse des contes de fées. Paris: Robert Laffont. 
Derisson, K. (2014). Blanche Neige et les sept nains, la création du chefd'œuvre de Walt Disney. Paris: L'Harmattan.

Finch, C. (1999). L'art de Walt Disney, de Mickey à Mulan. Paris: Editions de la Martinière.

Gaudreault, A \& Marion, P. (2013). La fin du cinéma ?. Paris: Armand Colin.

Grimm, J \& W. (2012). Blanche Neige et autres contes des frères Grimm. Paris: Pocket.

Holliss, R \& Sibley, B. (1987). Snow White and the Seven Dwarfs \& the making of the Classic Film. New York: Simon \&Schuster.

Johnston, O \& Thomas, F. (1995). Les méchants chez Walt Disney. Paris: Dreamland.

Kaufman, J-B. (2012). The fairest One of All. San Francisco: The Walt Disney Family Foundation Press.

Massuet, J-B. (2014). L’impact de la Performance Capture sur les théories du cinéma d'animation. Écranosphère, $n^{\circ} 1$.

Renaut, C. (1997). De Blanche Neige à Hercule, 28 longs métrages d'animation des studios Disney. Paris: Dreamland.

Smoodin, E. (2012). Snow White and the Seven Dwarfs. London: Palgrave macmillan, BFI.

\section{DVD}

Disney, W. (2009). Snow White and the Seven Dwarfs. Paris: Walt Disney Home Vidéo. 